Document downloaded from:

http://hdl.handle.net/10251/81901

This paper must be cited as:

Magin, I.; Polyakov, N.; Kruppa, Al.; Purtov, P.; Leshina, TV.; Kiryutin, AS.; Miranda Alonso, MÁ.... (2016). Low field photo-CIDNP in the intramolecular electron transfer in naproxenpyrrolidine dyads. Physical Chemistry Chemical Physics. 18(2):901-907. doi:10.1039/C5CP04233J.

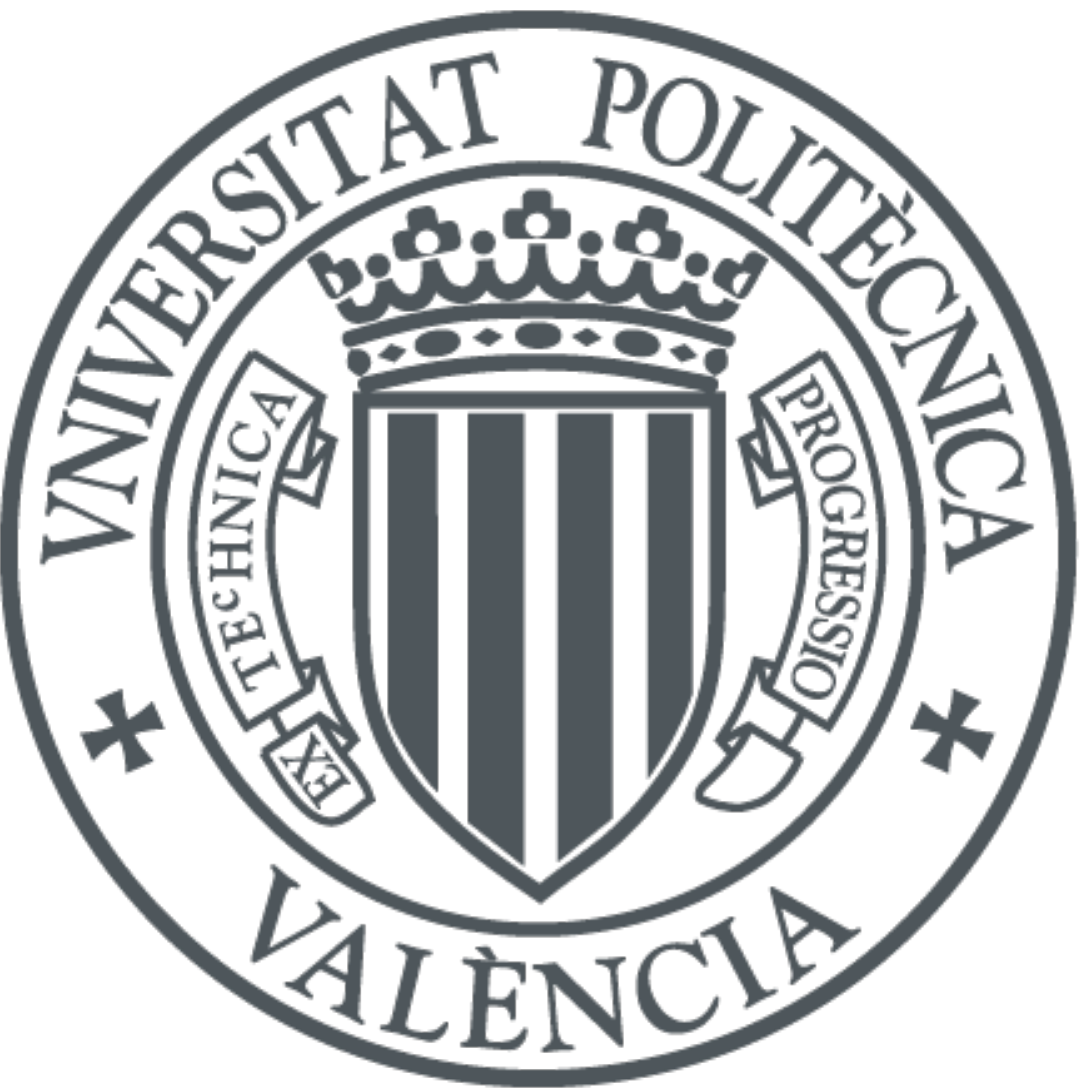

The final publication is available at http://doi.org/10.1039/c5cp04233j

Copyright Royal Society of Chemistry

Additional Information 


\title{
Low field photo-CIDNP in the intramolecular electron transfer of naproxen-pyrrolidine dyads
}

\author{
I. M. Magin ${ }^{*}$, N. E. Polyakov ${ }^{a}$, A. I. Kruppa ${ }^{a}$, P. A. Purtov ${ }^{a b}$, T. V. Leshina ${ }^{a}$, A. S. Kiryutin ${ }^{b}$, M. A. \\ Miranda ${ }^{d}$, E. Nuin ${ }^{d}$ and M. L. Marin ${ }^{d}$ \\ Institute of Chemical Kinetics and Combustion SB RAS, Institutskaya st. 3, 630090, Novosibirsk, Russia. E- \\ mail: magin@kinetics.nsc.ru
}

${ }^{b}$ Novosibirsk State University, 2, Pirogov st., 630090 Novosibirsk, Russia

International Tomography Center, SB RAS, Institutskaya 3-a, Novosibirsk 630090, Russia

¿Instituto Universitario Mixto de Tecnologia Quimica (UPV-CSIC), Universitat Politecnica de Valencia, Av. de los Naranjos s/n, E-46022, Valencia, Spain

Received 20th July 2015 , Accepted 12th November 2015

First published on the web 19th November 2015

Photoinduced processes with partial (exciplex) and full charge transfer in donor-acceptor systems are of interest because they are frequently used for modeling drug-protein binding. Low field photo-CIDNP (chemically induced dynamic nuclear polarization) for these processes in dyads, including the drug, $(S)$ - and $(R)$-naproxen and $(S)$ - $N$-methyl pyrrolidine in solutions with strong and weak permittivity have been measured. The dramatic influence of solvent permittivity on the field dependence of the $N$-methyl pyrrolidine ${ }^{1} \mathrm{H}$ CIDNP effects has been found. The field dependences of both $(R, S)$ - and $(S, S)$-dyads in a polar medium are the curves with a single extremum in the area of the $\mathrm{S}-\mathrm{T}_{+}$terms intersection. Moreover, the CIDNP field dependences of the same protons measured in a low polar medium present curves with several extrema. The shapes of the experimental CIDNP field dependence with two extrema have been described using the Green function approach for the calculation of the CIDNP effects in the system without electron exchange interactions. The article discusses the possible causes of the differences between the CIDNP field dependence detected in a low-permittivity solvent with the strong Coulomb interactions and in a polar solvent.

\section{Introduction}

Electron transfer (ET) is the most widespread and universal chemical process; therefore, ET attracts the permanent interest of researchers. 1 This interest extends in particular on photo-induced electron transfer (PET). PET processes in linked systems, dyads and triads, are often used as a model of enzyme-substrate interactions, as well as at the individual stages of photosynthesis. .23 Dyads are also utilized for the simulation of binding between medicine molecules and various transport proteins, because it is known that these processes often include donor-acceptor interactions. ${ }^{4}$ The main attention in such studies is paid to the detection of short-lived particles with partial and full charge transfer, namely, exciplexes and radical ion 
pairs (RIP), and clarification of the role of these particles. $\frac{5-7}{}$ Which is the first step in the chromophore excited state quenching? The formation of the exciplex, electron transfer or do these processes occur simultaneously. This issue has been widely discussed 2.6.7 in the investigation of the binding processes in biological systems, which involve the formation of intermediates with partial and full charge-transfer, including charge-transfer complexes and ion pairs...8Using photo-induced processes to simulate drug/receptor or drug/enzyme binding is based on the assumption that the donor-acceptor properties of the paramagnetic particles are not highly dependent on their generation pathway: photo-irradiation or thermal electron transfer. 2

One of the ways to study the connection between the exciplex and RIP is to analyze the effect of an external magnetic field (MFE) on time-resolved pulse photolysis or fluorescence.. .10

Another promising approach has been applied in the study of photo-induced processes involving partial (exciplex) and full (biradical ion) charge transfer in the dyad NPX-PYR $(S)$ - $N$-methyl-2-pyrrolidine $(R)$ or

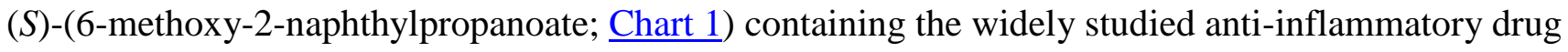
naproxen and $N$-methyl pyrrolidine using CIDNP and time-resolved fluorescence techniques. $\frac{11.12}{12}$

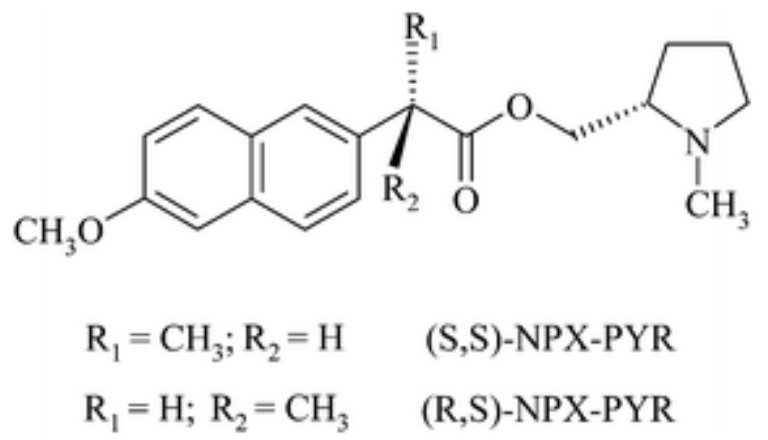

Chart $1(R, S)$ - and $(S, S)$-naproxen-pyrrolidine dyads.

The established reaction scheme includes the following short-lived intermediates: naproxen in the local excited state, exciplex and biradical ion (Scheme 1).

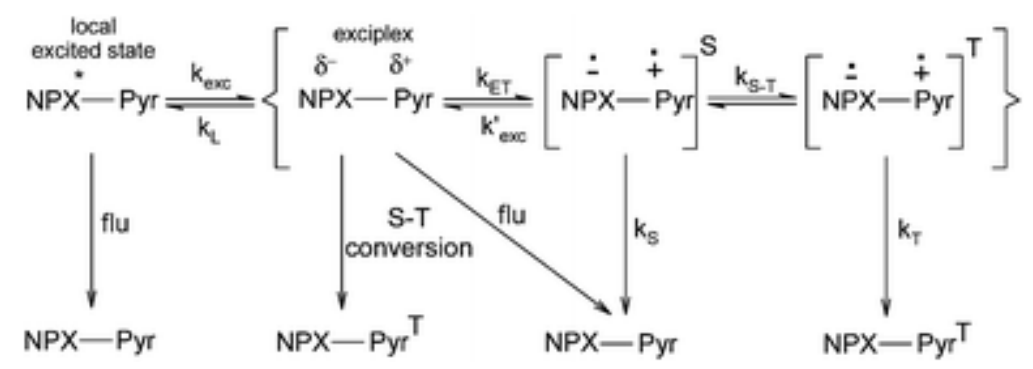

Scheme 1 Quenching of the excited singlet state of the NPX-Pyr dyad. The following symbols are used to describe the rate constants of separate stages: $k_{\mathrm{exc}}-$ local excited state transfer to exciplex; $k_{\mathrm{L}}-$ transfer of exciplex to local excited state; $k_{\mathrm{ET}}$ - electron transfer; $k_{\mathrm{exc}}{ }^{\prime}-$ transfer from biradical ion to exciplex; $k_{\mathrm{S}-\mathrm{T}}-$ singlet-triplet conversion in biradical ion; flu - fluorescence quenching; $k_{\mathrm{S}}$ and $k_{\mathrm{T}}-$ back electron transfer from the singlet and triplet spin states of biradical ion.

Analysis of the kinetics of fluorescence quenching allows one to get the rate constant of the separate stages. Therefore, in a solution with $\varepsilon=8.1, k_{\text {exc }}{ }^{\prime}=2.0 \times 10^{8} \mathrm{~s}^{-1}$ and $1.2 \times 10^{8} \mathrm{~s}^{-1} ; k_{\mathrm{ET}}=0.23 \times 10^{8} \mathrm{~s}^{-1}$ and 0.34 $\times 10^{8} \mathrm{~s}^{-1}$ was obtained for the $(R, S)$ - and $(S, S)$-enantiomers of the NPX-Pyr dyad. In a polar solvent $(\varepsilon=$ $37.5), k_{\text {exc }}{ }^{\prime}=2.6 \times 10^{8} \mathrm{~s}^{-1}$ and $2.0 \times 10^{8} \mathrm{~s}^{-1} ; k_{\mathrm{ET}}=1.29 \times 10^{8} \mathrm{~s}^{-1}$ and $1.62 \times 10^{8} \mathrm{~s}^{-1}$ was obtained for the $(R, S)$ and $(S, S)$-enantiomers.

The states with partial (exciplex) and full charge transfer are, according to the CIDNP data, in a rapid dynamic equilibrium that is in the nanosecond range. 12

It seems interesting to study the CIDNP effects in the NPX-PYR dyads in low magnetic fields. Because the chemical polarization is a differential effect, unlike the integral MFE, one would expect a significant 
diversity in the CIDNP field dependence in the media with different permittivity due to the change in the exciplex/biradical ion ratio, as well as in the Coulomb interactions. Moreover, the abovementioned fast balance between the states with partial and full charge transfer in the naproxen-pyrrolidine dyad lets us expect the possibility of the impact of the rapid exchange between the exciplex and biradical ion on the spin evolution.

It should be noted that the influence of the exciplex/biradical ion balance on the recombination probability of the radical ion pair in the external magnetic field has been considered recently by G. Grampp with co-workers. $?$ The authors take this into account as a reduction in the concentration of the radical ion pairs all along the transfer of the system to the exciplex state.

We can expect the sensitivity of the low field CIDNP to the Coulomb interactions strength due to the dependence of the CIDNP intensity on the paramagnetic precursors lifetime in the zone of proximity between the paramagnetic centers, wherein $S$ and $T_{+,-}$terms intercross.

The influence of these parameters on the width and location of the extremum on the field dependence of the biradical recombination probability has been shown earlier theoretically using density matrix formalism. ${ }^{13}$ In the present study, this theoretical approach has been applied for the discussion of the key factors that affect the CIDNP in the NPX-PYR dyad in a low magnetic field.

\section{Experimental section}

The $(R, S)$ - and $(S, S)$-NPX-PYR dyads have been synthesized as described elsewhere. ${ }^{4}$ Naphthalene (Aldrich) was sublimated prior to use. Triethylamine (TEA) was distilled from zinc powder. Acetonitrile$\mathrm{d}_{3}(\mathrm{ACN}, \mathrm{D} 99.9 \%)$ and benzene- $\mathrm{d}_{6}$ (BZ, D99.8\%) (both from Sigma-Aldrich) have been used in the NMR and CIDNP experiments. The solvent permittivity was varied by changing the proportion of a BZ/ACN mixture. The mixture of $0.7 \mathrm{~mL}$ of $\mathrm{ACN}(\varepsilon=37.5)$ and $2.4 \mathrm{~mL}$ of BZ $(\varepsilon=2.3)$ has a dielectric constant of about $10 . \frac{14.15}{}$ All samples were bubbled with $\mathrm{N}_{2}$ for $15 \mathrm{~min}$ to remove any dissolved oxygen just before photolysis. The concentration of the dyad was varied from 0.2 to $2 \mathrm{mM}$.

The ${ }^{1} \mathrm{H}$ NMR and CIDNP spectra were obtained at room temperature on a homemade NMR spectrometer (300 MHz ${ }^{1} \mathrm{H}$ operating frequency, $\left(\tau\left(90^{\circ}\right)=3.5 \mu \mathrm{s}\right)$ ) equipped with an Oxford $7 \mathrm{~T}$ cryomagnet. ${ }^{16}$ The samples in $5 \mathrm{~mm}$ quartz tubes were irradiated directly in the probe of the NMR spectrometer via a flexible liquid optical light guide with a $90^{\circ}$ prism on its top (20 pulses, $50 \mathrm{~Hz}, 308 \mathrm{~nm}$ ).

A field-cycling unit with fast digital positioning of a high-resolution nuclear magnetic resonance probe in a spatially varying magnetic field $\stackrel{16}{ }$ was used to measure the CIDNP spectra. The field-cycling setup allows one to vary the magnetic field between $0.1 \mathrm{mT}$ and $7 \mathrm{~T}$ and detect the NMR spectra under sample rotation keeping a high resolution of $0.5 \mathrm{~Hz}$. This was based on the step-motor-driven transfer of the whole NMR probe along the bore axis of the $7 \mathrm{~T}$ cryomagnet of the NMR spectrometer down to an electromagnet (Helmholtz pair) located in the stray field.

The CIDNP effects are the signals with increased absorption or emission in the NMR spectra of the products obtained from the radical reactions. An analysis of these effects, described elsewhere, provides

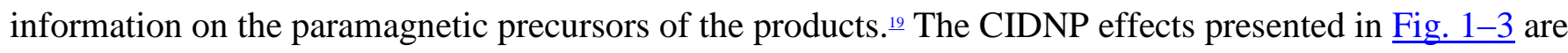
the difference in the intensity of the polarized lines detected during UV irradiation of the dyad solutions and a related system comprised naphthalene/triethylamine (TEA), and the intensity of the lines in the NMR spectra obtained without irradiation. 


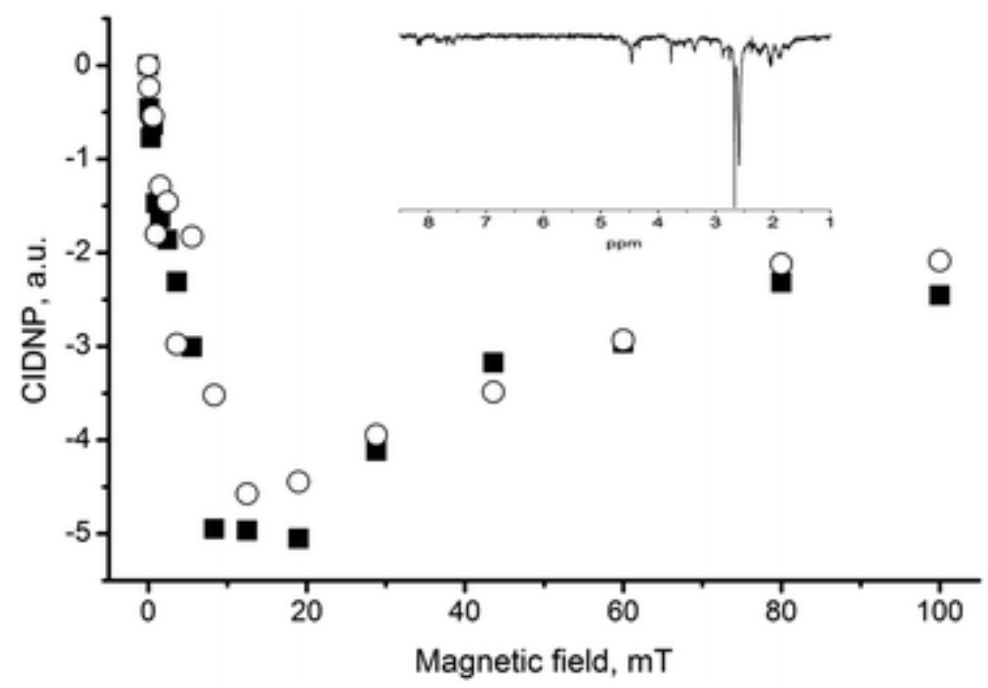

Fig. 1 CIDNP field dependence (low field region) for $\mathrm{N}-\mathrm{CH}_{3}$ protons $(2.6 \mathrm{ppm})$ measured after laser irradiation $(308 \mathrm{~nm})$ of $2 \mathrm{mM}$ solutions of the $(R, S)-(\square)$ and $(S, S)$-NPX-PYR (O) dyads in ACN $(\varepsilon=37.5)$. The inset shows the CIDNP effects of $N$-methyl pyrrolidine protons in the $(S, S)$-NPX-PYR dyad detected in a magnetic field of $12.5 \mathrm{mT}$.

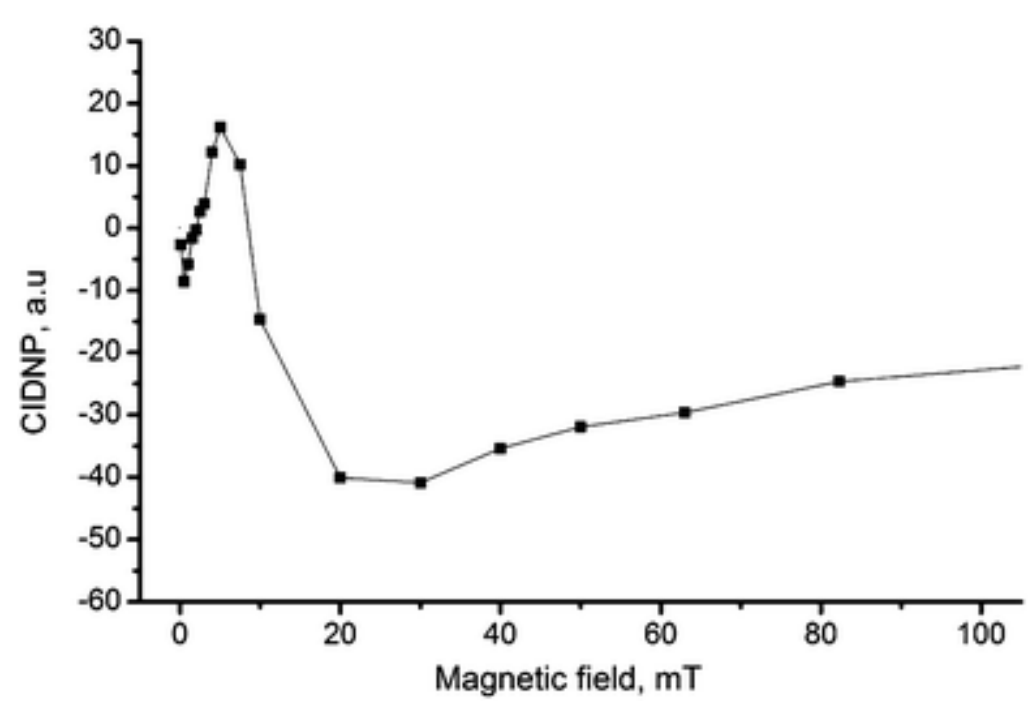

Fig. 2 CIDNP field dependence for $\mathrm{N}-\mathrm{CH}_{3}$ protons measured after laser irradiation $(308 \mathrm{~nm})$ of $2 \mathrm{mM}$ solution of the $(R, S)$-NPX-PYR dyad in a ACN/BZ mixture at $\varepsilon=10$.

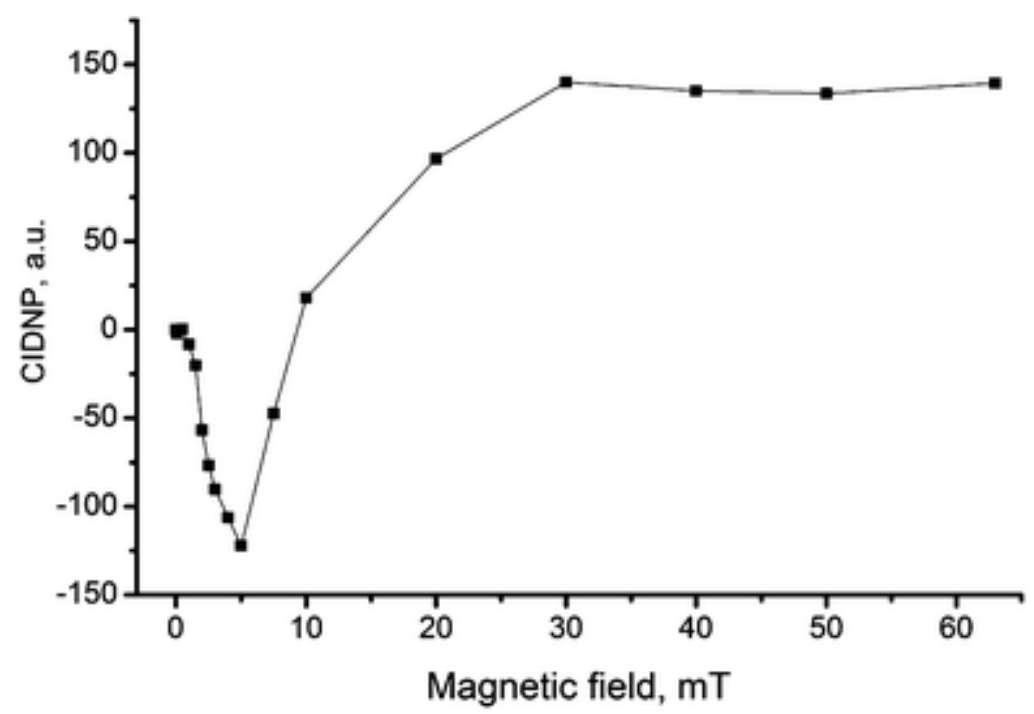

Fig. 3 CIDNP field dependence (low field region) for $\mathrm{N}-\mathrm{CH}_{2}$ protons of TEA measured after laser irradiation $(308 \mathrm{~nm})$ of $2 \mathrm{mM}$ solution of naphthalene in the presence of $10 \mathrm{mM}$ TEA in $\mathrm{CD}_{3} \mathrm{CN}$. 


\section{Results and discussion}

\section{CIDNP field dependence in the NPX-PYR dyad and the related system comprised naphthalene + triethylamine}

The CIDNP field dependence for $(R, S)$ - and $(S, S)$-isomers of the dyad (Chart 1) has been measured in the magnetic field from 0 to $100 \mathrm{mT}$ with two different solvent permittivities (in acetonitrile, $\varepsilon_{1}=37.5$ and in a $\mathrm{ACN} / \mathrm{BZ}$ mixture, $\varepsilon_{2}=10$ ). These two different permittivity values have been specifically chosen based on the analysis of the high field CIDNP dependence on the solvent dielectric constant and the exciplex fluorescence quantum yields. They correspond to the maximum and minimum contribution of the exciplex in the chemical polarization formed in a biradical ion. .10 .11

Strong polarization has been observed for the $\mathrm{CH}, \mathrm{CH}_{2}$, and $\mathrm{CH}_{3}$ protons in the $\mathrm{N}$-methyl-pyrrolidine fragment (emission) and very weak emission for the aromatic protons. The CIDNP field dependence of the $\mathrm{N}-\mathrm{CH}_{3}$ protons in the $(R, S)$-NPX-PYR dyad are shown in Fig. 1 and 2. The CIDNP effects of the $\mathrm{CH}$ and $\mathrm{CH}_{2}$ protons have similar field dependences.

The CIDNP field dependence of the $\mathrm{N}-\mathrm{CH}_{3}$ protons in the NPX-Pyr dyads in the low permittivity region demonstrates the changes of sign (Fig. 2).

For comparison we have measured the low field CIDNP in a related system comprised naphthalene/TEA. Note that the donor and acceptor redox parameters in the quenching reaction of the naphthalene excited singlet state by TEA are similar to those found in the NPX-PYR dyad.17.18

The low field CIDNP dependence of the $\mathrm{N}-\mathrm{CH}_{2}$ protons of TEA is shown in Fig. 3. This dependence is almost a mirror image of that for the dyad protons in a low polar environment (Fig. 2). Both these dependences have maxima at about $5 \mathrm{mT}$ and $25 \mathrm{mT}$.

Note that the chemical polarization for all these three field dependences is formed in the biradical ion or RIP with similar magnetic resonance parameters (the HFI constants for the $\mathrm{CH}_{2}$ and $\mathrm{CH}$ protons of the methyl-pyrrolidine radical cation are about 2.9-5.8 $\mathrm{mT}$, for the $\mathrm{CH}_{3}$ protons it is about $2.9 \mathrm{mT}$, and the HFI constants for the $\mathrm{CH}_{2}$ protons of the TEA radical cation are $2.19 \mathrm{mT}^{19.20}$ ).

We can suggest that the different signs of the extrema in the CIDNP field dependence of the dyad protons at weak permittivity and the $\mathrm{TEA} \mathrm{CH}_{2}$ protons at high permittivity are a result of the difference in the ratio of the rate constants of back electron transfer (BET) from the singlet and triplet spin states of the RIP and biradical ion, $k_{\mathrm{S}}$ and $k_{\mathrm{T}}$ (see Scheme 1). There are reference data, which show that $k_{\mathrm{T}}$ is higher than $k_{\mathrm{S}}$ in the act of BET in the RIP of "naphthalene/TEA" in a polar environment. 21 The prevalence of the opposite sign of CIDNP in the NPX-Pyr dyad has been previously explained by the contribution to the value of $k_{\mathrm{S}}$ from the additional channel of dyad excitation quenching. ${ }^{12}$ This channel is a fast reversible transition between the biradical ion and singlet exciplex.

A comparison of the field dependence extrema positions with the HFI constants values in the paramagnetic precursors of the polarized products allows us to draw some conclusions. Therefore, the CIDNP field dependence of the NPX-Pyr dyads in ACN, which has one extremum whose position exceeds the magnitude of HFI constant twice, includes the contribution from the electron exchange interaction $(J)$. On the contrary, both dependences with several extrema (Fig. 2 and 3) are similar to those that are usually observed for products of RP without sizeable $J .19$

Next, we provide the theoretical analysis of the field dependences to explain the dramatic difference between the dyads field dependences in media with high and weak permittivity, and to explain why the CIDNP field dependence of the dyad in the conditions of strong Coulomb interactions (weak permittivity) 
was similar to that in the product of diffusive quenching in a polar solution, which does not include the electron exchange interaction.

\section{CIDNP field dependence theoretical description}

For the theoretical description of the CIDNP effects in the dyad we have been applied the Green's functions method. This approach is widely used to describe the magnetic and spin effects in radical reactions. ${ }^{22.23} \mathrm{In}$ particular, a modified version has been successfully used for the theoretical description of the spin effects (CIDNP, magnetic effect) in biradicals. .18 In this approach, the recombination probability or CIDNP effects are described through the spin and molecular dynamics of the radical centers.

The recombination probability of pairs is given by a spin state vector $\hat{w}$ :

$$
\hat{w}=\hat{U}(1+\hat{g} \hat{U})^{-1} \hat{g} \rho_{0}
$$

The matrix $\hat{U}$ describes the recombination rate constant of a particular spin state; the matrix $\hat{g}$ describes the spin dynamics of the radical centers averaged relative movement and the residence time of a pair in the reaction zone; $\rho_{0}$ is the initial density matrix of the pair.

When calculating the CIDNP effects we are considering only one flip flop transition of the electron and nuclear spins. The recombination probability of the singlet state at the start from the singlet in this case is described by formula (2):

$$
w^{\mathrm{S}}=\frac{U_{0} \tau_{\mathrm{S}}}{1+U_{0} \tau_{\mathrm{S}}}
$$

where $U_{0}$ is a quasi-monomolecular constant of the recombination rate and $\tau_{\mathrm{s}}$ is the residence time of a pair in the reaction zone in the singlet state. The recombination probability in the singlet state in an initial triplet state can be written as (3):

$$
w^{\mathrm{T}}=\frac{1}{3} \frac{U_{0}\left(\tau_{\mathrm{r}}-\tau_{\mathrm{S}}\right)}{1+U_{0} \tau_{\mathrm{S}}}
$$

where $\tau_{\mathrm{r}}$ is the total residence time of the pair in the reaction zone.

It is known that the change in the reaction state from singlet to the triplet state results in the changing of only the sign of the CIDNP but not the shape of the field dependence. ${ }^{24}$ For the purposes of this study, this fact is crucial because as described above, both the singlet and triplet spin states of the biradical ion participate in the quenching of the dyad's excited state. 11

Fig. 4 shows the calculated CIDNP field dependences in the case when recombination proceeds simultaneously from the singlet and triplet spin states of radical pair (RP). Herein, the electron exchange interaction is not included in the calculation (Fig. 4). 


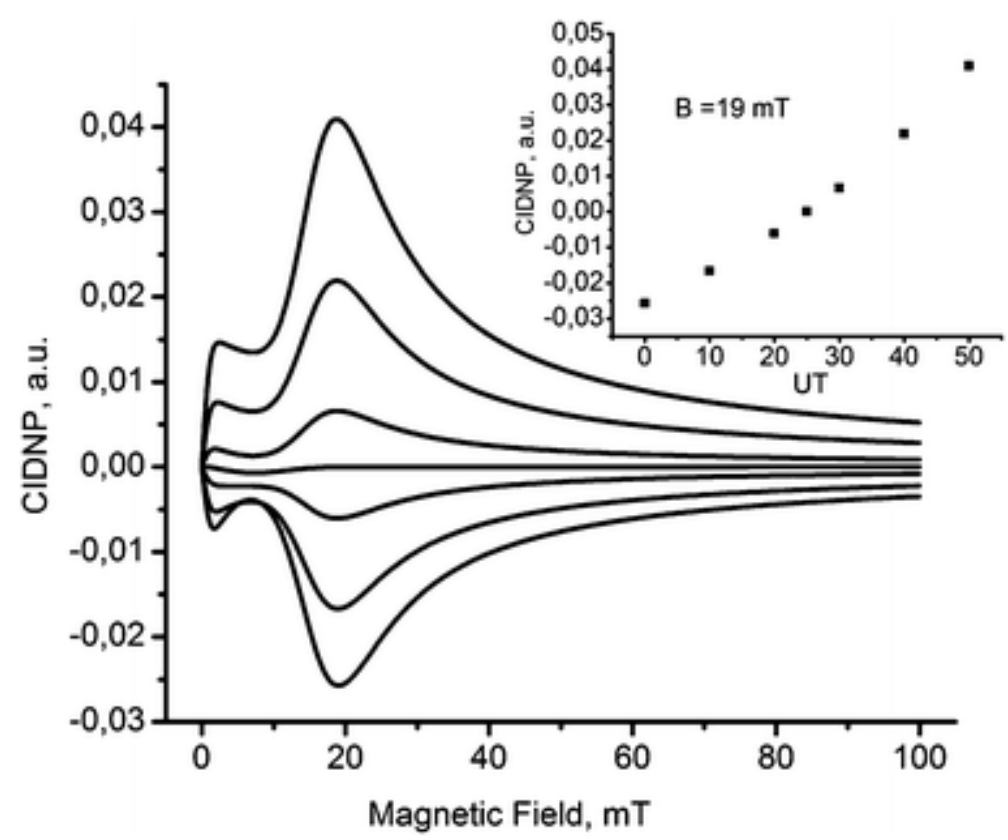

Fig. 4 Calculated CIDNP field dependence for RP with one magnetic nucleus with spin $I=3 / 2$ for different contributions of recombination from the singlet and triplet states $\left(U_{\mathrm{S}}+U_{\mathrm{T}}\right) \tau_{\mathrm{r}}=0.5$. From the bottom to top $-U_{\mathrm{T}} \tau_{\mathrm{r}}=0,0.1,0.2,0.25,0.3,0.4,0.5$. Inset - the value for CIDNP at a magnetic field of $19 \mathrm{mT}$ from the contribution of the triplet recombination channel $\left(U_{\mathrm{T}} \tau_{\mathrm{r}}\right)$.

As can be observed from Fig. 4, the position of the extremum in the field dependence of the CIDNP practically does not shift and the contributions from the recombination channels can be considered as additive. Therefore, in further calculations we will consider that the reaction only proceeds from any one state.

To simulate the experimental field dependences shown in Fig. 2 and 3 , we have used the following simplification: instead of the biradical ion containing many magnetic nuclei, we have calculated a radical pair with one nucleus with spin $I$.

The calculations shown in Fig. 5 are made for the diffusion motion of radicals. The initial spin state of the RP and the reaction state have been assumed as a singlet. Note that the alterations in the CIDNP sign are characteristic for RP with $I \geq 3 / 2$. A similar pattern has been obtained for the exponential model of the radical's relative motion under the condition that the RP's lifetime is large enough. .5

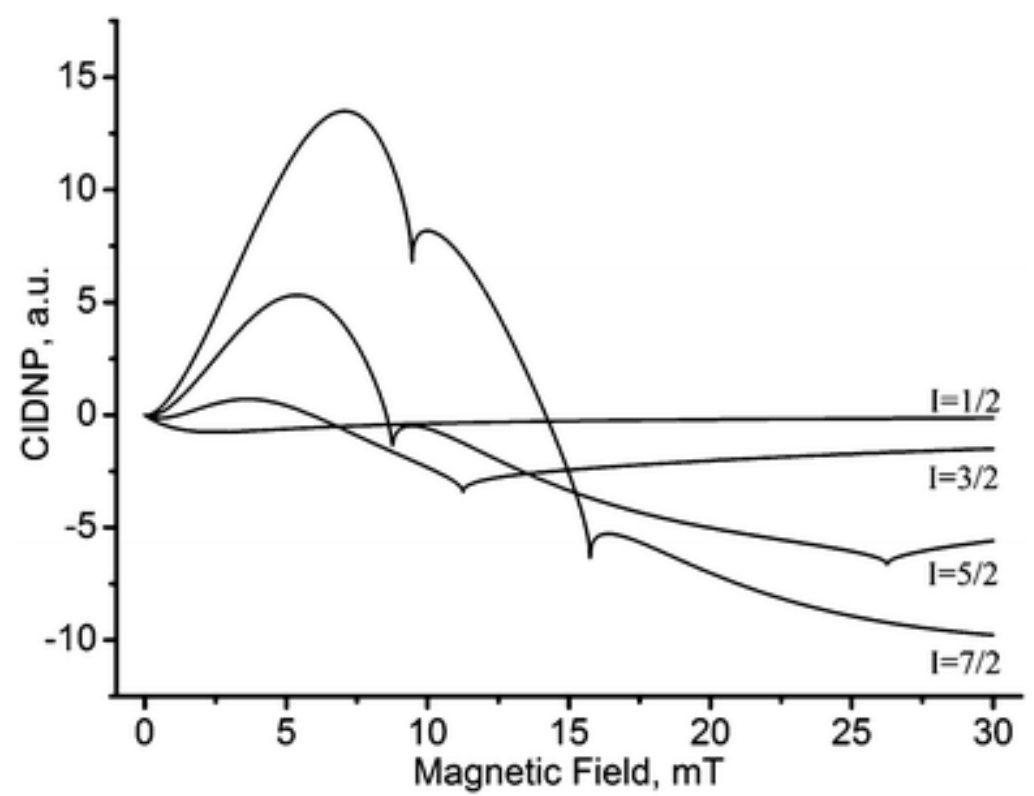

Fig. 5 Calculated dependence of CIDNP for the product from RPs with different values of nuclear spin $I$ on 
Similar field dependences can also be obtained under the following assumptions. An ensemble of RP containing six equivalent protons is equivalent to 4 subensambles of RP, which have magnetic nuclei with spins $I=3,2,1,0$. Fig. 6 shows the field dependence of the CIDNP for RP containing 6 equivalent protons.

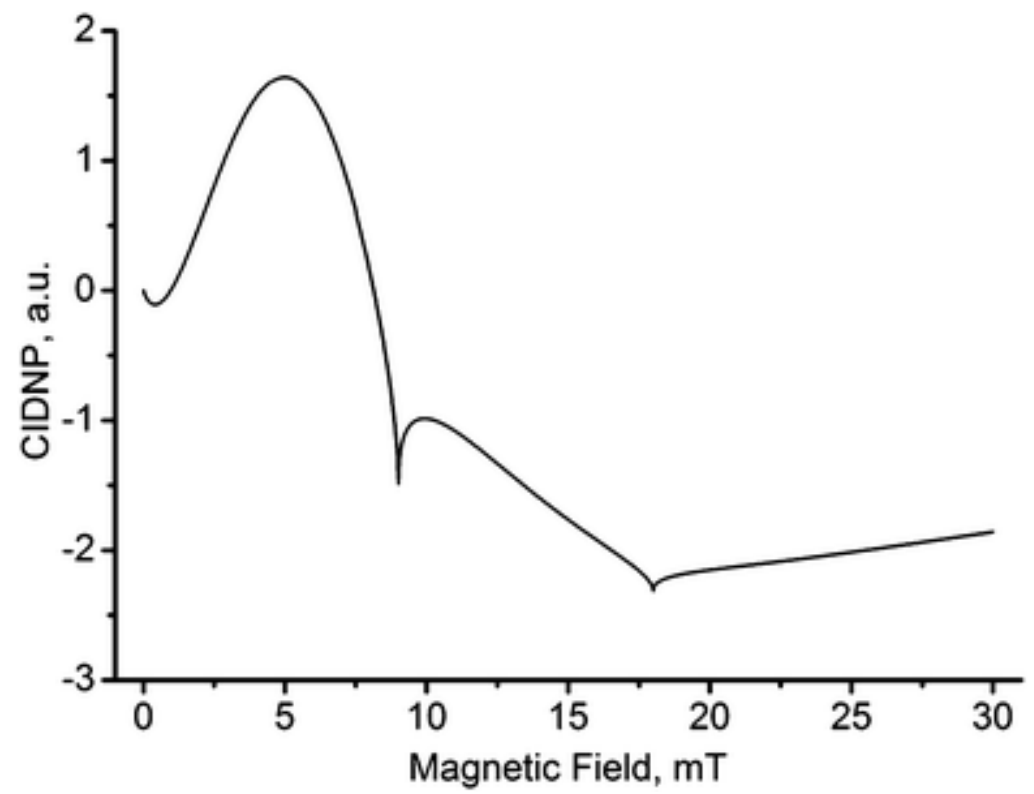

Fig. 6 Calculated dependence of CIDNP of the product from RP containing 6 equivalent protons on the magnetic field strength at a HFI constant $a=3 \mathrm{mT}$.

The shape of the CIDNP field dependences in Fig. 5 and 6 is in a qualitative agreement with the experimental dependence registered for the protons of the dyad in a solvent with weak permittivity (Fig. 2), as well as for CIDNP detected in the diffusion quenching of excitation in the related system (Fig. 3). For RPs that contain one magnetic nuclei with $I=3$ (or six equivalent protons), the first extremum is predicted in the fields $B \approx 2 a$ and the second one with an opposite sign in the field $B \approx 6 a$. The experimental field dependences show extrema located at $5 \mathrm{mT}$ and 25-30 mT (Fig. 2 and 3 ).

The same field dependence was also predicted by the well-known semi-classical approximation for these radical pairs. ${ }^{26}$ This is the case when the effective constant HFI ( $\left.a_{\text {eff }}\right)$ of the detected radical significantly exceeds $a_{\text {eff }}$ of the partner radical. The polarized NMR signal in the studied interval of magnetic fields involves all the alpha $\mathrm{CH}_{3}, \mathrm{CH}_{2}$ and $\mathrm{CH}$ protons in the $\mathrm{N}$-methyl pyrrolidine radical cation in which the HFI constants vary in the range of $2.9-5.8 \mathrm{mT},{ }_{19}$ and the partner radical, which is the substituted naphthalene radical anion, has $a_{\text {eff }} \sim 0.5 \mathrm{mT} .27$

Thus, a comparison of the theory with our experimental data shows that the CIDNP in the linked system in a low-polar medium (Fig. 2) can be described under the assumption of a strong electron exchange interaction during contact and zero electron exchange interaction between contacts, as in the case of the separated radical pair in high-polar media (Fig. 3). To understand why in the linked system, in a low-polar solvent wherein the Coulomb interactions may play an essential role, the contribution from the electron exchange interaction does not manifest itself, we consider two specific regions wherein the singlet-triplet transitions occur in a low magnetic field (Fig. 7). 

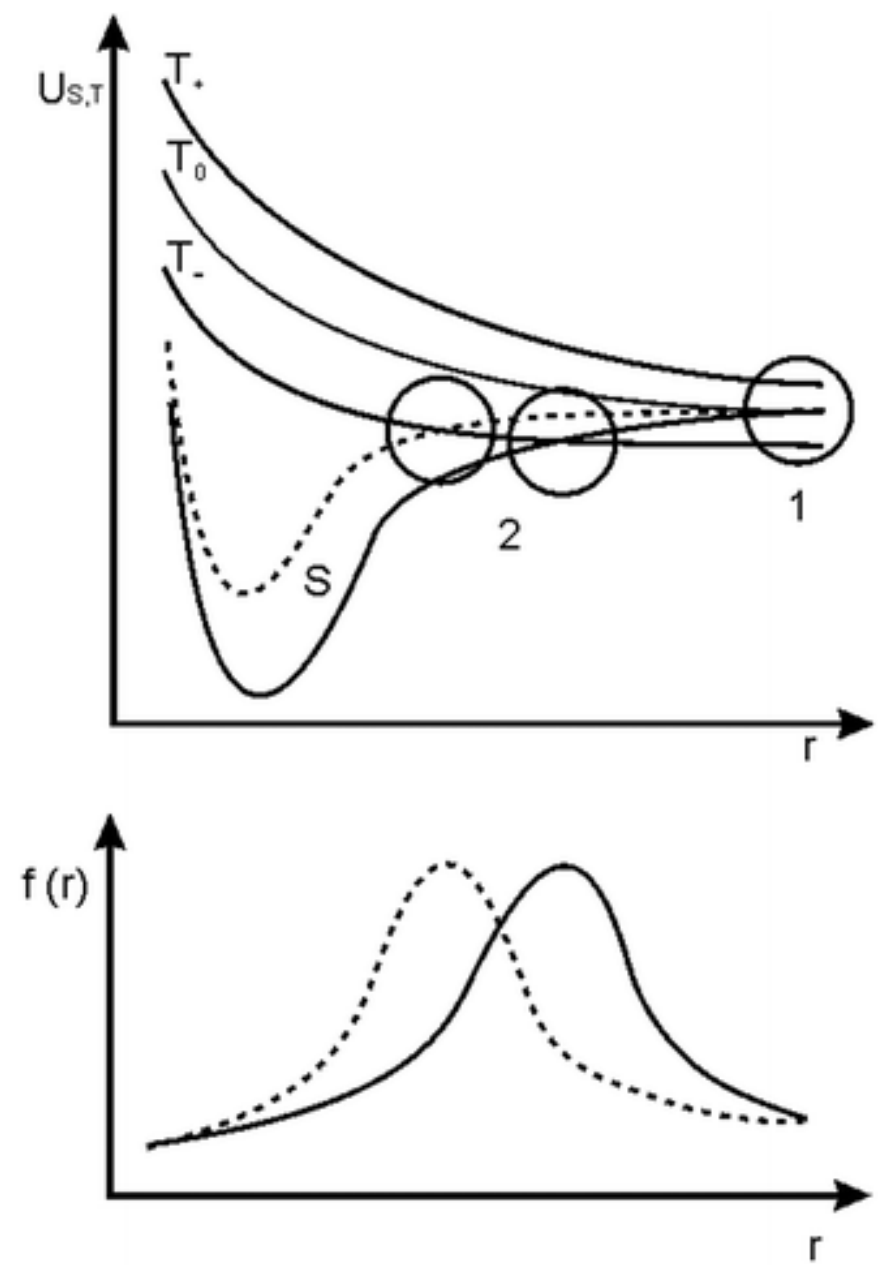

Fig. 7 At the top - the dependence of the singlet and triplet energy levels of the biradical on the distance between the radical centers. The two transition zones 1 and 2 correspond to the area of weak and the strong exchange interaction, respectively. At the bottom - the probability of the distance distribution between the radical centers in the biradical at different permittivities: the solid line corresponds to high permittivity and dotted line refers to low permittivity.

As is well known, these two regions play key roles in CIDNP formation (Fig. 7). In region (1) the singlet and triplet terms are close to each other and the electronic exchange interaction is usually insignificant. In region (2) the intersection of the singlet with one of the triplet terms $\left(\mathrm{T}_{+}\right.$or $\mathrm{T}_{-}$depending on the sign of the exchange interaction) is situated. In separated radical pairs, the formation of chemical polarization usually occurs in region (1). In region (2) these pairs are typically located for a very short period of time; therefore, this region has no contribution to the CIDNP. Nevertheless, there are many systems wherein the main contribution to the polarization comes from region (2). Among them a typical example is the biradicals derived from cyclic ketones. ${ }^{28.29}$ The relative motion of the radical centers in these biradicals is determined by the dynamic behavior of the polymethylene chain, namely, its conformational transitions. The S-T transition probabilities and the distance distribution between the radical centers in different conformations are determined by the corresponding potential barriers and the changes in total energy. When the centers are very close the probability is small, further it grows and reaches a maximum at relatively large distances and then decreases again. The qualitative shape of the dependence is shown in Fig. 7.

The CIDNP would have the maximum in a magnetic field, wherein the intersection of the $S$ and $T_{ \pm}$terms coincides with the maximum of the distribution function $\left(f_{\mathrm{r}}\right)$. Region (1), wherein the singlet and triplet terms are close to each other, usually makes a small contribution to the biradical polarization. However, in some cases, the situation may be changed dramatically. For example, in the study of the CIDNP in a strong magnetic field $(4.7 \mathrm{~T})$ region (1), on the contrary, is the main source of the polarization, because the 
effective transition in the area of terms intersection becomes small due to the strong increase in the slope of terms intersection..$^{30}$

The change between the contribution to the CIDNP from regions 1 and 2 in the biradical ion can also occur in the presence of acceptors. $\frac{31}{}$ The influence of the acceptor on the S-T evolution is described in detail in the RP theory and has been studied experimentally. ${ }^{32.33}$ Basically, it comes down to a reduction in the lifetime of RP. In the case of biradicals, the acceptors action leads to a reduction in the residence time of the biradical in both regions 1 and 2. In accordance with the general consideration of the prevailing influence of acceptors on region 1 would be expected: it is known that in free radical reactions a decrease in the CIDNP from distant trajectories occurs primarily. However, the experimentally observed shift in the field dependence extremum to the strong field shows that in the case of biradicals the situation is different. This fact can be explained if we take into account that the residence times of the biradical in regions 1 and 2 are different. In the region of intersection in region (2), the system spends less time than in region (1) wherein the exchange interaction was much smaller than the HFI. The CIDNP effects in region 1 for long lived RP, to which most biradicals belong, are often averaged: case $(A \cdot \tau \geq 1)$. In this case, shortening the lifetime can lead to growth in the CIDNP due to the decrease in the $(A \cdot \tau)$ value. At the same time, the chemical polarization arising in region 2 can only decrease. Thus, the result of the acceptor influence on the field dependence of biradical described in ref. 26 is a manifestation of the CIDNP formed in region 1, where electron exchange interaction is negligible.

Some insight into the contribution to the observed CIDNP from regions 1 and 2 can be obtained from the example of the CIDNP in the NPX-PYR dyad (Fig. 8). This figure compares the scale of the effects in weak and strong fields $(4.7 \mathrm{~T})$ in solutions with a high and low dielectric constant. The figure shows that for field dependence obtained in a solution with high $\varepsilon$ the region of $\mathrm{S}-\mathrm{T}_{+}$crossing indeed makes a significant contribution to the CIDNP, whereas in the field dependence for weak permittivity the contribution from region 1 prevails.

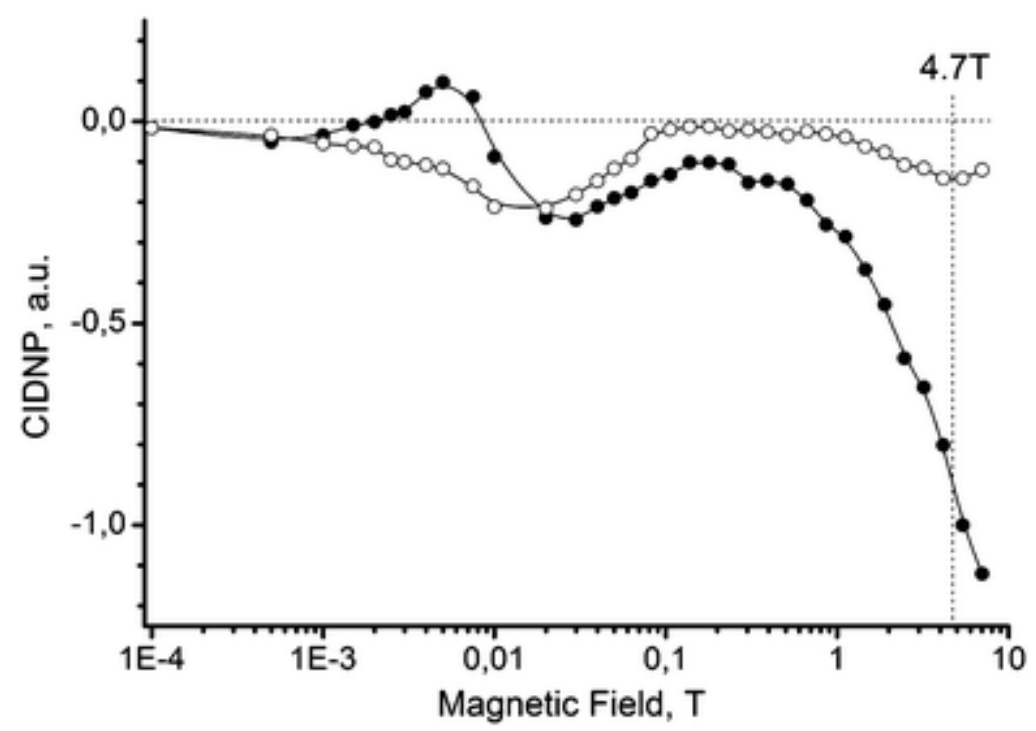

Fig. 8 CIDNP field dependence of $\mathrm{N}_{-} \mathrm{CH}_{3}$ protons measured after laser irradiation (308 $\mathrm{nm}$ ) of $2 \mathrm{mM}$ solution of the $(R, S)$-NPX-PYR dyad in $\mathrm{ACN}(\circ, \varepsilon=37.5)$ and a $\mathrm{ACN} / \mathrm{BZ}$ mixture $(\bullet, \varepsilon=10)$ on the external magnetic field strength $(B)$. The ratio of field dependence at $4.7 \mathrm{~T}$ corresponds to the literature values reported for the CIDNP dependence on solvent permittivity. 11

It is worth noting that a similar situation also occurs in the optically detected EPR spectra and MARY spectra in non-polar systems..$^{34}$ In this case for the theoretical description of the magnetic field influence on the recombination of charged particles generated by radiolysis in the Coulomb field hyperfine interaction is 
always sufficient, i.e. the exchange interaction does not manifest itself due to the short time that the system spends in the term crossing region 2.

In accordance with the image considered above, it seems that the appearance of the CIDNP from zone " 1 " in the field dependence of the NPX-PYR dyad in a low polar solvent is also possible for several reasons. This is due to the Coulomb attraction between the paramagnetic centers of the dyad and the fast exchange of the biradical ion with the exciplex. It is also possible to expect that this exchange will be manifested in a low polar environment, wherein the contribution from the exciplex is maximum. 12

Therefore, upon decreasing the permittivity, the strength of the Coulomb attraction, which strongly influences the position of the maximum of the distribution function, will increase and the maximum of the distribution function in Fig. 7 has to be shifted to shorter distances between the radical centers. This would result in a shift of the extremum in the CIDNP field dependence to higher magnetic fields.

However, the slope of the intersection of the terms is increased at shorter distances and therefore the probability of transitions in the area of intersection of the terms decreases (Fig. 7). The CIDNP intensity in this case can be significantly reduced.

Even more important is the increase in the probability of the BET with decreasing distance between the radical centers. The acceleration of BET can lead to the destruction or the severe reduction of the CIDNP due to a decrease in the S-T evolution time. It was shown that in the NPX-Pyr dyads the dynamic equilibrium between the biradical ion and exciplex significantly accelerates the BET from its singlet spin state. 12

According to the study, ${ }^{10}$ it occurs due to the decay of the exciplex through fluorescence, intrinsic singlettriplet conversion and the transfer to the local excited state. All these processes also accelerate the biradical ion decay ( $\underline{\text { Scheme 1)}}$.

Because the rates of the transitions between the states with partial and full charge transfer are in the nanosecond range, one can expect that the fast exchange between the exciplex and the biradical ion would influence S-T evolution. In this case, the transfer of the system into the exciplex and its return to the biradical ion would be accompanied by the loss of spin correlation in the state with full charge transfer. ${ }^{32.33 .35}$ This results in a reduction of the contribution to CIDNP from the near trajectories (region 2, Fig. 7) and leads to the predominance of the CIDNP from region 1 formed at long distances.

These considerations are fully confirmed by the theoretical description of the experimental field dependence of CIDNP in the NPX-Pyr dyad in a low-polar solvent (Fig. 2) that did not take into account the electron exchange interaction.

Finally, the CIDNP field dependence for the $(R, S)$ - and $(S, S)$-diastereoisomers of the NPX-PYR dyad in a polar medium (ACN, Fig. 1) contain only one extremum with a negative sign.

The origin of the CIDNP extremum for the $(S, S)$ - and $(R, S)$-diastereomers are located at magnetic fields between 12 and $20 \mathrm{mT}$ (Fig. 1), which are several times greater than the HFI. It can be explained by the intersection of the $\mathrm{S}$ and $\mathrm{T}_{+}$terms with a moderate electron exchange interaction between the paramagnetic centers in the biradical ion. According to Kaptein rules, ${ }^{24}$ the negative sign of the CIDNP with the singlet precursor multiplicity will correspond to the positive sign of the electron exchange interaction.

Thus, it has been demonstrated that the low field CIDNP dependence on the magnetic field strength varies greatly depending on the solvent permittivity and the exciplex quantum yields.

\section{Conclusions}


This study shows that the difference in molecular and spin dynamics in a linked system in the media of strong and weak permittivity leads to dramatic differences in the CIDNP field dependence in low magnetic fields. The CIDNP effects in these fields demonstrate a high sensitivity to the Coulomb interactions and the rapid dynamic equilibrium between states with partial (exciplex) and complete charge transfer (biradical ion).

\section{Acknowledgements}

This study was supported by the grant 14-03-00-192 of the Russian Foundation of Basic Research. The authors are also deeply grateful to Professor Hans-Martin Vieth for the given opportunity to conduct experiments on his unique equipment.

\section{References}

1. S. Y. Reece and D. G. Nocera, Annu. Rev. Biochem., 2009, 78, 673 CrossRef CAS PubMed.

2. S. Richert, A. Rosspeinter, S. Landgraf, G. Grampp, E. Vauthey and D. R. Kattnig, J. Am. Chem. Soc., 2013, 135, 15144 CrossRef CAS PubMed.

3. S. Aich and S. Basu, J. Phys. Chem., 1998, 102, 722 CrossRef CAS.

4. I. Vaya, R. Perez-Ruiz, V. Lhiabet-Vallet, M. C. Jimenez and M. A. Miranda, Chem. Phys. Lett., 2010, 486, 147 CrossRef CAS.

5. U. Werner and H. Staerk, J. Phys. Chem., 1995, 99, 248 CrossRef CAS.

6. D. R. Kattnig, A. Rosspeintner and G. Grampp, Angew. Chem., 2008, 47, 960 CrossRef CAS PubMed.

7. D. R. Kattnig, A. Rosspeintner and G. Grampp, Phys. Chem. Chem. Phys., 2011, 13, 3446 RSC.

8. I. Vaya, V. Lhiabet-Vallet, M. C. Jimenez and M. A. Miranda, Chem. Soc. Rev., 2014, 43, 4102 RSC.

9. N. E. Polyakov, M. B. Taraban and T. V. Leshina, Photochem. Photobiol., 2004, 80, 565 CrossRef CAS PubMed.

10. H. Cao, Y. Fujiwara, T. Haino, Y. Fukazawa, C.-H. Tung and Y. Tanimoto, Bull. Chem. Soc. Jpn., 1996, 69, 2801 CrossRef CAS.

11. I. M. Magin, N. E. Polyakov, E. A. Khramtsova, A. I. Kruppa, Yu. P. Tsentalovich, T. V. Leshina, M. A. Miranda, E. Nuin and M. L. Marin, Chem. Phys. Lett., 2011, 516, 51 CrossRef CAS.

12. E. A. Khramtsova, V. F. Plyusnin, I. M. Magin, A. I. Kruppa, N. E. Polyakov, T. V. Leshina, E. Nuin, M. L. Marin and M. A. Miranda, J. Phys. Chem. B, 2013, 117, 16206 CrossRef CAS PubMed.

13. I. M. Magin, P. A. Purtov, A. I. Kruppa and T. V. Leshina, J. Phys. Chem. A, 2005, 109, 7396 CrossRef CAS PubMed.

14. V. Subramanian, B. S. Bellubbi and J. Sobhanadri, Pramana J. Phys., 1993, 41, 9 CrossRef CAS.

15. B. Acemioglu, M. Arik, H. Efeoglu and Y. Onganer, THEOCHEM, 2001, 548, 165 CrossRef CAS.

16. S. Grosse, F. Gubaydullin, H. Scheelken, H.-M. Vieth and A. V. Yurkovskaya, Appl. Magn. Reson., 1999, 17, 211 CrossRef CAS.

17. I. M. Magin, N. E. Polyakov, E. A. Khramtsova, A. I. Kruppa, A. A. Stepanov, P. A. Purtov, T. V. Leshina, Y. P. Tsentalovich, M. A. Miranda, E. Nuin and M. L. Marin, Appl. Magn. Reson., 2011, 41, 205 CrossRef CAS. 
18. C. K. Mann and K. K. Barnes, Electrochemical Reactions in Nonaqueous Systems, M. Dekker, New York, 1970 Search PubMed.

19. N. S. Landolt-Bornstein, Numerical Data and Functional Relationship in Science and Technology: Magnetic Properties of Free Radicals, Springer-Verlag, Berlin, 1988 Search PubMed.

20. V. M. Grigoryants, O. A. Anisimov and Yu. N. Molin, J. Struct. Chem., 1982, 23(N3), 327 CrossRef.

21. J. Bargon, J. Am. Chem. Soc., 1977, 99, 8350 CrossRef CAS.

22. P. A. Purtov and A. B. Doktorov, Chem. Phys., 1993, 178, 47 CrossRef CAS.

23. P. A. Purtov, A. B. Doktorov and A. V. Popov, Chem. Phys., 1994, 182, 149 CrossRef CAS.

24. K. M. Salikhov, Yu. N. Molin, R. Z. Sagdeev and A. L. Buchachenko, in Spin Polarization and Magnetic Field Effects in Radical, ed. Yu. N. Molin, Akademiai Kiado, Budapest, 1984 Search PubMed.

25. S. A. Sukhenko, P. A. Purtov and K. M. Salikhov, Khimicheskaja Physika, 1983, 1, 21 Search PubMed (in Russian).

26. N. E. Polyakov, P. A. Purtov, T. V. Leshina, K. M. Salikhov and R. Z. Sagdeev, Chem. Phys. Lett., 1986, 129, 357 CrossRef.

27. M. Shiotani, L. Sjoeqvist, A. Lund, S. Lunell, L. Eriksson and M. B. Huang, J. Phys. Chem., 1990, 94, 8081 CrossRef CAS.

28. F. J. J. De Kanter, J. A. den Holander, A. H. Huiser and R. Kaptein, Mol. Phys., 1977, 34, 857 CrossRef CAS.

29. F. J. J. De Kanter, R. Kaptein and R. A. von Santen, Chem. Phys. Lett., 1977, 45, 575 CrossRef CAS.

30. Yu. P. Tsentalovich, A. V. Yurkovskaya, A. A. Obynochny, R. Z. Sagdeev, P. A. Purtov and A. A. Shargorodsky, Chem. Phys., 1989, 139, 307 CrossRef CAS.

31. A. V. Popov, P. A. Purtov and A. V. Yurkovskaya, Chem. Phys., 2000, 252, 83 CrossRef CAS.

32. I. M. Magin, V. S. Shevel'kov, A. A. Obynochny, A. I. Kruppa and T. V. Leshina, Chem. Phys. Lett., 2002, 357, 351 CrossRef CAS.

33. K. Schulten and P. G. Wolynes, J. Chem. Phys., 1978, 68, 3292 CrossRef CAS.

34. E. V. Kalneus, D. V. Stass and Yu. N. Molin, Appl. Magn. Reson., 2005, 28(3-4), 213 CrossRef CAS.

35. A. I. Kruppa, T. V. Leshina, R. Z. Sagdeev, E. C. Korolenko and N. V. Shokhirev, Chem. Phys., 1987, 114, 95 CrossRef CAS. 